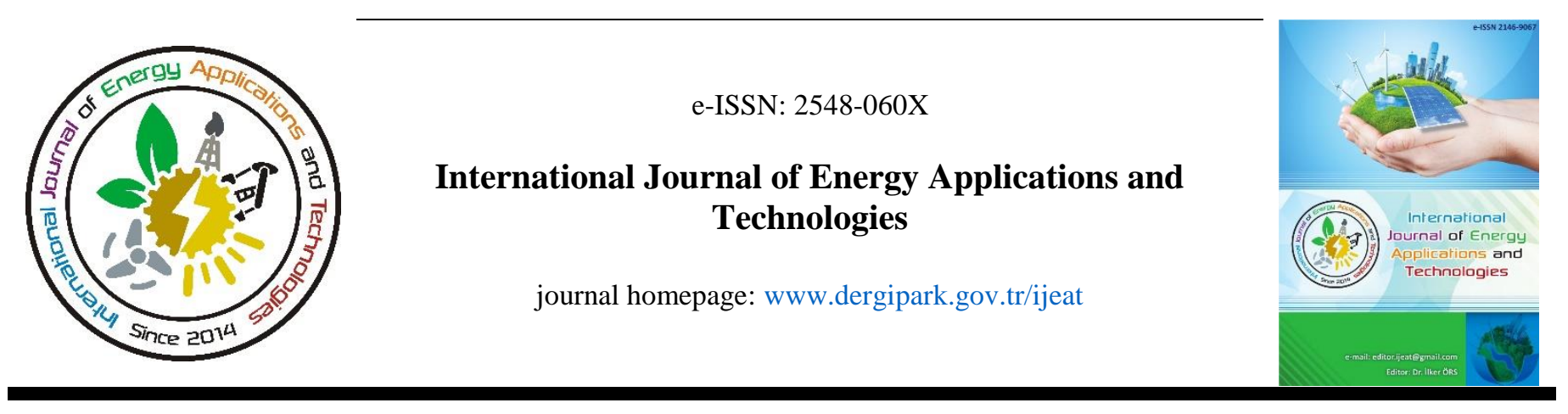

Original Research Article

\title{
Smart metering field implementation with power line communication in low voltage distribution grid
}

\author{
Murat Şen ${ }^{1 *}$, Seda Üstün Ercan ${ }^{2}$ \\ ${ }^{\text {I} E l e c t r o n i c s ~ a n d ~ A u t o m a t i o n ~ D e p a r t m e n t, ~ Y e s ̧ i l y u r t ~ D e m i r ~ C ̧ e l i k ~ V o c a t i o n a l ~ S c h o o l, ~ O n d o k u z ~ M a y l s ~ U n i v e r s i t y, ~ S a m s u n, ~ T u r k e y ~}$ \\ ${ }^{2}$ Electrical and Electronics Engineering Department, Faculty of Engineering, Ondokuz Mayls University, Samsun, Turkey
}

\author{
ARTICLE INFO \\ * Corresponding author \\ murat.sen@omu.edu.tr \\ Received September 29, 2020 \\ Accepted February 9, 2021 \\ Published by Editorial Board \\ Members of IJEAT \\ (C) This article is distributed by \\ Turk Journal Park System under \\ the CC 4.0 terms and conditions. \\ doi: 10.31593/ijeat.801513
}

\begin{abstract}
Smart Grids (SG) enable power generation, distribution, transmission, customers and utilities to transfer, predict, monitor and manage energy usage effectively. In order to provide them, SGs need to be fully realized by integrating communication technologies infrastructures. Today, SGs have some main systems. These are Advanced Meter Reading (AMR), distributed renewable energy, energy storage, electric vehicle and smart city-home implementations. AMR is the most prominent SG component between all SG main systems. Because it provides to follow all consumers remotely and momentarily using their Smart Meters (SM). AMR system is based on SM, gateway, meter data management software and wired or wireless communication method. Nowadays, Power Line Communication (PLC) is the most popular wired communication method for remote meter reading because of try to use existing electric distribution grid infrastructure. On the other hand, GPRS/EDGE/3G is one of the widespread wireless communication method for remote meter reading implementations but its costly and external dependence has started the search for new communication methods instead. In this paper, practically twelve electric meters are tried to be read remotely with PLC in Yeşilırmak Electric Distribution Company field until the distribution transformer. These remote meter reading results that captured with PLC will evaluate in AMR software. Then an AMR system model is put forward thanks to this field implementation. Beside this a hybrid communication method has been suggested for smart metering. PLC key parameters will find out. In addition, data concentrator (DC), meter and gateway that has PLC features equipment field configurations will research.
\end{abstract}

Keywords: AMI; AMR; PLC; Smart grid; Smart metering

\section{Introduction}

SG has many definition but its general feature is two way communications between consumer and grid. Some SG events that monitoring, protection, automation, integration are included two way communications with utility companies and end users [1]. This bi-directional communication is provided by Smart Metering process so Smart Metering is one of the key process because of its crucial role in terms of SG. SM is quite different from classical energy meter. It doesn't measure only total consumption or time depended register value. SM is an advanced energy meter that can measure real time consumption, current, voltage, energy outage, active-reactive power, harmonics, power factor, demand and frequency [2]. Beside this SM can save these informations in its memory and when the utility companies need these added informations, SM send these informations from field to the meter data management software because of its remote communication capability. Thus, SM provides to instantly monitor distribution grid. Distribution companies catch a one more way to manage distribution grid easier.

SM, communication methods and SM data management software constitute Automated Meter Reading (AMR) 
System. These aspects take part in Advanced Metering Infrastructure (AMI) System at the same time. AMI has some complex processes according to AMR. AMI has to use Smart Metering results for automated billing, remote reconnectiondisconnection, real time pricing, theft detection, volt/var management and system integrations so AMI can be evaluated developed version of AMR [3].

The communication methods can be classified as wired and wireless technologies in terms of generation, transmission, distribution operations of SG [4]. Wired communications technologies are commonly Power Line Communications (PLC), Fiber Optic cables networks and Digital Subscriber Line (DSL/Ethernet) while wireless technologies are generally GPRS/EDGE/3G/4.5G/5G, Radio Frequency (RFRF Mesh) Wi-Fi, Wi-Max, Bluetooth, Zigbee, LoRaWan [20].

The communication technologies have some advantages and disadvantages, However, the aim of all modern and future communications methods is able to provide high rate data communication, low signal degradation, high scalability, better networking infrastructure, high coverage, substantiality, low cost, high authentication, strong encryption system and hybrid operation adequacy features, so advanced SG requirements are ensured for communication [5].

The communication methods have an important role for Smart Metering. Because measured data are sent to the Smart Metering software with communication methods from the field. If the communication is stuck, all measured SM data flow will be stop from the field to Smart Metering software. PLC, GPRS/EDGE/3G, RF-RF Mesh communication technologies generally come to the forefront in remote meter reading implementations. Main role belongs to electric distribution grid in PLC method and electric distribution grid is already exist peer to peer on the field so it can take additional role like communication between SMs without any new installation issue. Therefore, PLC method research should be done in deeply.

Last times PLC was one of the most popular communication method that captured research interest for Smart Metering and it is still succeed to be catchy research area [6]. Beside this it can be used with other communication models like GPRS/EDGE/3G as hybrid in low voltage distribution grid. This study includes GPRS/EDGE/3G - PLC hybrid communication model, mainly PLC.

\section{Power Line Communication Method}

Power Line Communication (PLC) is a technology that carries measured data of SG via existing underground cabling and overhead lines in generation, distribution and transmission network. Generally, these measured SG data which was carried by PLC, includes remote meter reading process namely AMR, AMI applications [7].

PLC has strong features to manage end to end communication beside this it has demonstrated to be beneficial way with available for free network in communication of SG applications [8].

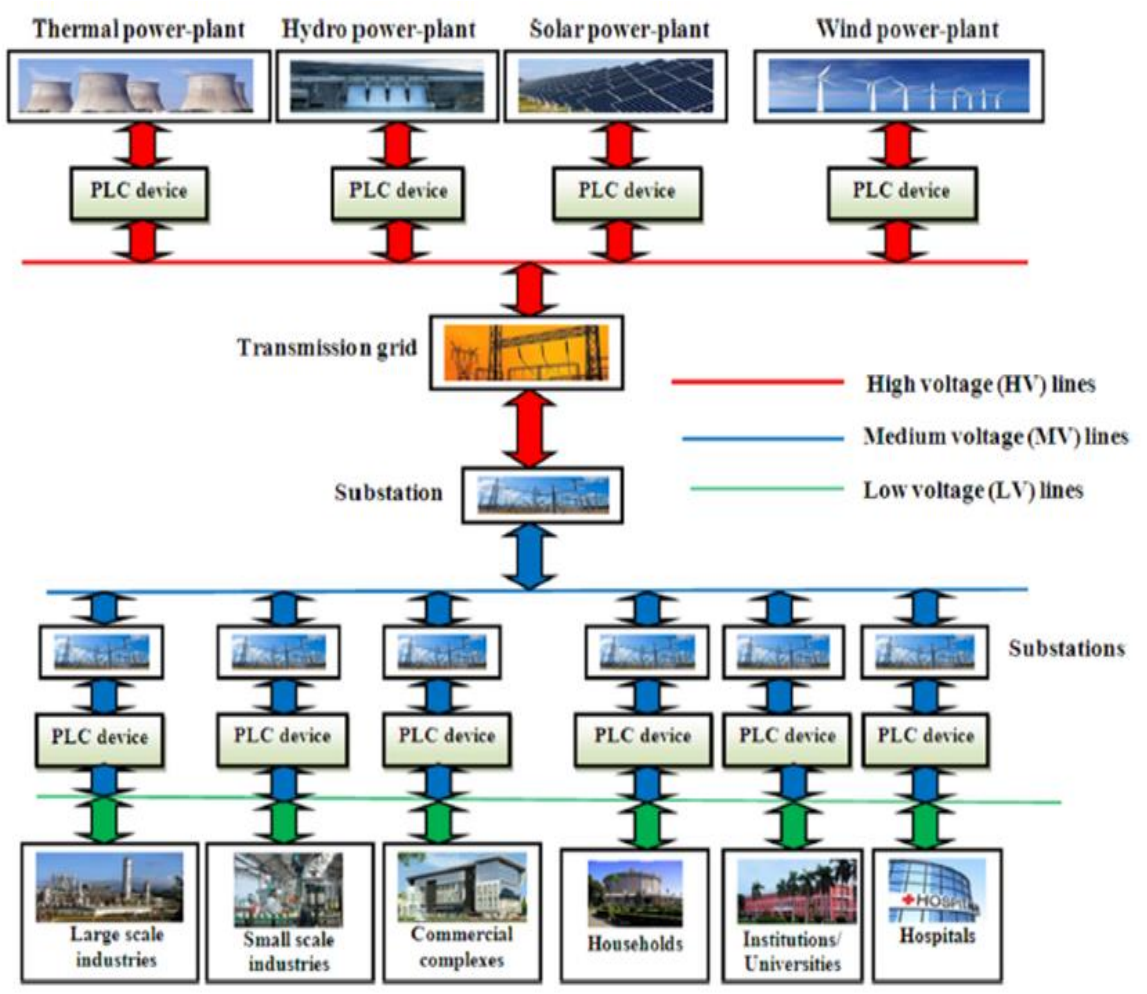

Fig. 1. Power line communications system architecture in SG [8] 
When the current electricity grid is considered PLC has some advantages and disadvantages. Usage of existing grid is easy way for implementations. Because there is no need installation process so time gain is obtained and all devices which will communicate are in same grid. There isn't any antenna and tuning process beside this communication is possible hard field situations that can describe deep underground lines, metal-shielded cable, there is no coverage problem [8]. PLC doesn't need third party company for communication by comparison GPRS/EDGE/3G so it can eliminate addiction to another partner for communication and this situation is good at in terms of cyber security. On the other hand, existing electricity grid primarily is not designed for communication purpose. Communication impedance and communication channel conditions are not constant in order to electricity is alive and its load, equipment position (like circuit breakers), voltage, current, power factor, power can change easily. Signal attenuation may occur for long line because of carried data may be lost [19].

Although PLC is mainly used for remote meter reading process in terms of AMR, AMI in low voltage grid, it can see other SG implementations for communication purpose. These implementations are renewable energy, general lightning, electric vehicle charging process, smart home.

PLC has some classification and standardization. They include frequency intervals, data rate. According to SG implementations suitable way is preferred. There is some independent or government regulator to define PLC standards. European Committee for Electrotechnical Standardization (CENELEC), European Committee for Standardization (CEN) and European Telecommunications Standards Institute (ETSI) are responsible electric engineering establishment to define PLC standards in Europe. The establishments roles are, determine the frequency intervals, transmitted signal limits for PLC $[9,18]$.

\subsection{PLC classifications}

Modulation techniques, classifications and standardizations are crucial for a PLC signal. PLC signal behaviors depend on these parameters. PLC signals are classified with four topics, these are Ultra-narrow band power line communication (UNB-PLC), Narrow band power line communication (NBPLC), Quasi band power line communication (QB-PLC), Broad band power line communication (BB-PLC) [8]. UNBPLC provides $30-300 \mathrm{~Hz}$, frequency range and 100-120 Bps data rate. QB-PLC consists of NB-PLC and BB-PLC. It provides advantages about frequency band as 1-10 $\mathrm{MHz}$ and its data speed can reach $2 \mathrm{Mbps}$. BB-PLC frequency range is nearly between $1.8 \mathrm{Mhz}-86 \mathrm{MHz}$ and it has high very data speed in short range so BB-PLC specially use smart home applications. BB-PLC is expected to replace with wireless communication system [8].

NB-PLC is the most useful class in terms of SG applications, especially AMR, AMI implementations. NB-PLC covers many PLC modulation techniques and it operates up to 500 $\mathrm{kHz}$ frequency band. NB-PLC divided frequency intervals by CENELEC according to usage area in Europe. A-band range is $3-95 \mathrm{kHz}$ for distribution utility to control and low voltage network. B-band range is $95 \mathrm{kHz}-125 \mathrm{kHz}$ is used by end users without any access protocol. C-band is $125 \mathrm{kHz}-140$ $\mathrm{kHz}$ is used for home networking systems with obligatory access protocol. D band is $140 \mathrm{kHz}-148.5 \mathrm{kHz}$ for alarm and security systems without any access protocol [9]. In this case CENELEC-A band should consider for remote meter reading implementations in NB-PLC for Europe. NB-PLC has widespread usage area for communication in electricity grid. These usage areas and explanations are shown with Fig. 2. in terms of transmission and distribution.

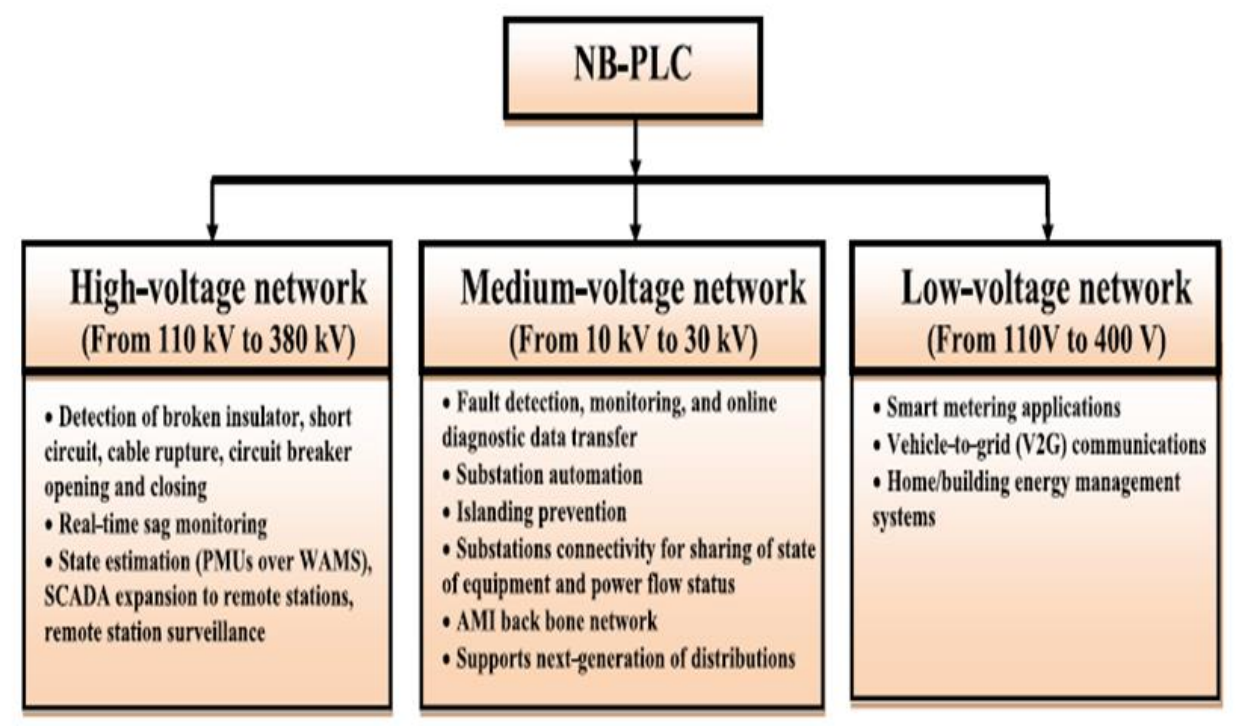

Fig. 2. NB-PLC usage area in SG [8] 


\subsection{PLC standardization}

Powerline Intelligent Metering Evolution (PRIME) (ITU-T G.9904), G3-PLC (ITU-T G.9903), IEEE 1901.2-2013, ITUT.G.hnem are developed famous standards by some alliance for NB-PLC [8]. Among these standards PRIME and G3PLC come into prominence for Smart Metering according to others because of their high field implementations. Both standards use OFDM (Orthogonal Frequency Division Multiplexing) methods for modulation in order to increase date rate and provide high robustness. Main difference is subcarriers numbers between G3-PLC and PRIME. While PRIME OFDM symbols have 97 subcarriers, G3-PLC OFDM symbols have 36 subcarriers [9].

PRIME and G3-PLC Technologies have capability to pass through electricity grid assets and demonstrated to be beneficial and feasible for advanced distribution automation process (like AMI, AMR), which is one of the main application of SG infrastructure [8]. PLC classes, standards and modulation techniques are illustrated with their frequency ranges and data rates by Fig. 3 .

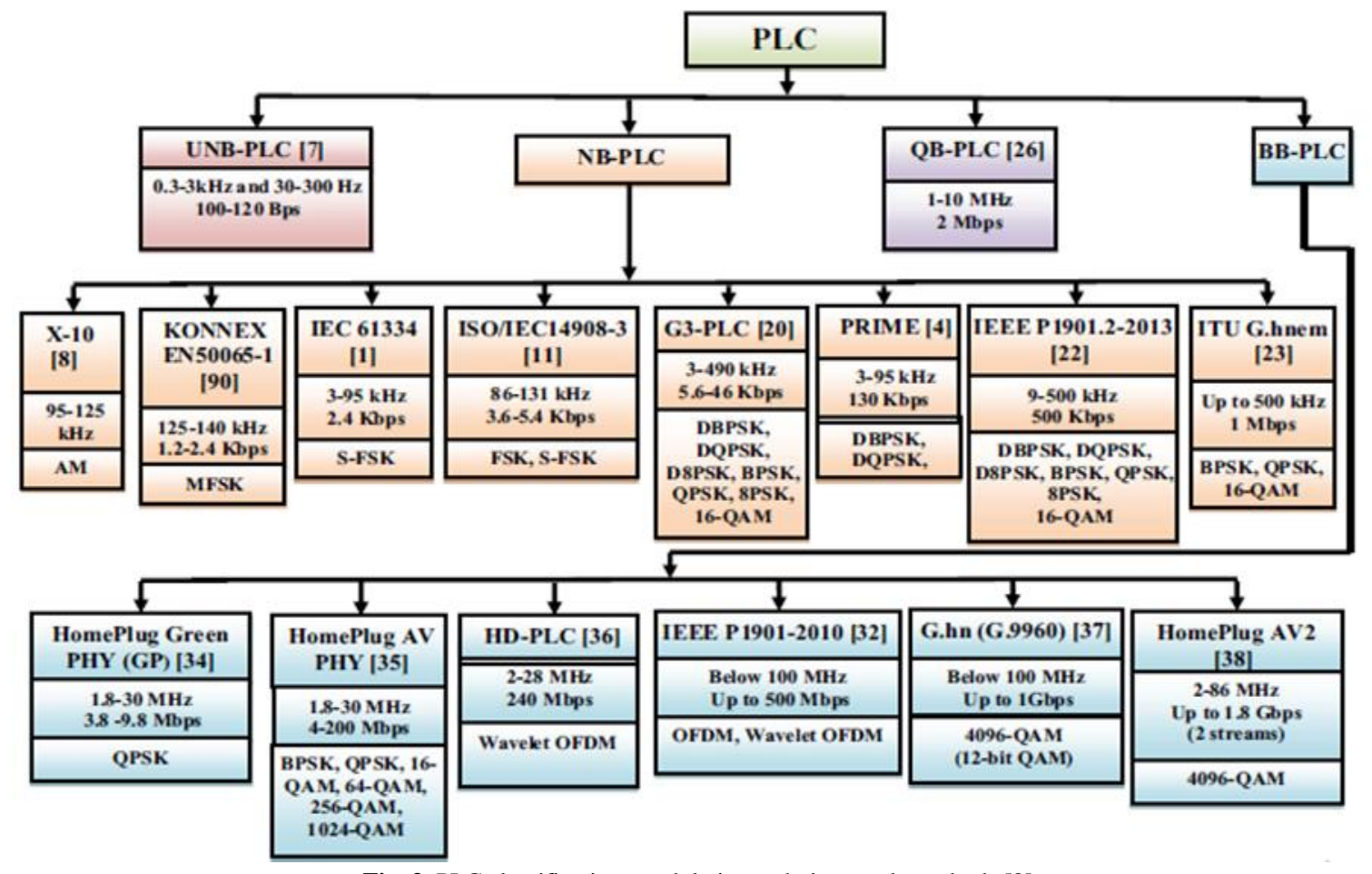

Fig. 3. PLC classification, modulation technique and standards [8]

\section{General Model of Smart Metering with Power Line Communication}

NB-PLC can play significant role to reach low voltage grid users and distribution operators. NB-PLC, actually, has the main advantages of very low cost because of its simplify of installation, since the grid is already available (i.e., the power line), and the lack of a service provider cost, since the utilities are normally owners of the grid.

One of the main SG implementations based on NB-PLC that is already widely spread in many countries is the AMI. The AMI which uses NB-PLC physical main parts are SMs and DC. The PLC gateway may be included in the meter or externally connected via communication port RS 485, RS 232. Thus, these combinations generate SM that use PLC as communication method. SM install to the consumers area. On the other hand, DC installs to the low voltage side of distribution transformers and DC defines the SM which are feed same distribution transformer and collect SM measurements to transmit the AMI software. The DC periodically capture SM data over NB-PLC, aggregate the measurements and send them to AMI software using cellular network [10]. This cellular network is generally GPRS/EDGE/3G between DC and AMI software. GPRS/EDGE/3G can be preferred for devices that need to transmit a lot of data and communicate as a point to point. The measurement devices that use GPRS/EDGE/3G are able to communicate directly central access server of AMI software [11]. Thus, it is the most beneficial solution to use GPRS/EDGE/3G communication way only on the DC side where is in low voltage grid part of distribution transformer. Basic structure is shown about usage of PLC for Smart Metering in distribution grid in Fig. 4. While SMs where install to the consumer in LV distribution grid, use PLC to transmit the measured data, DC that collects and aggregates SMs data, uses GPRS to send the collected SMs data to the AMI software [12]. 


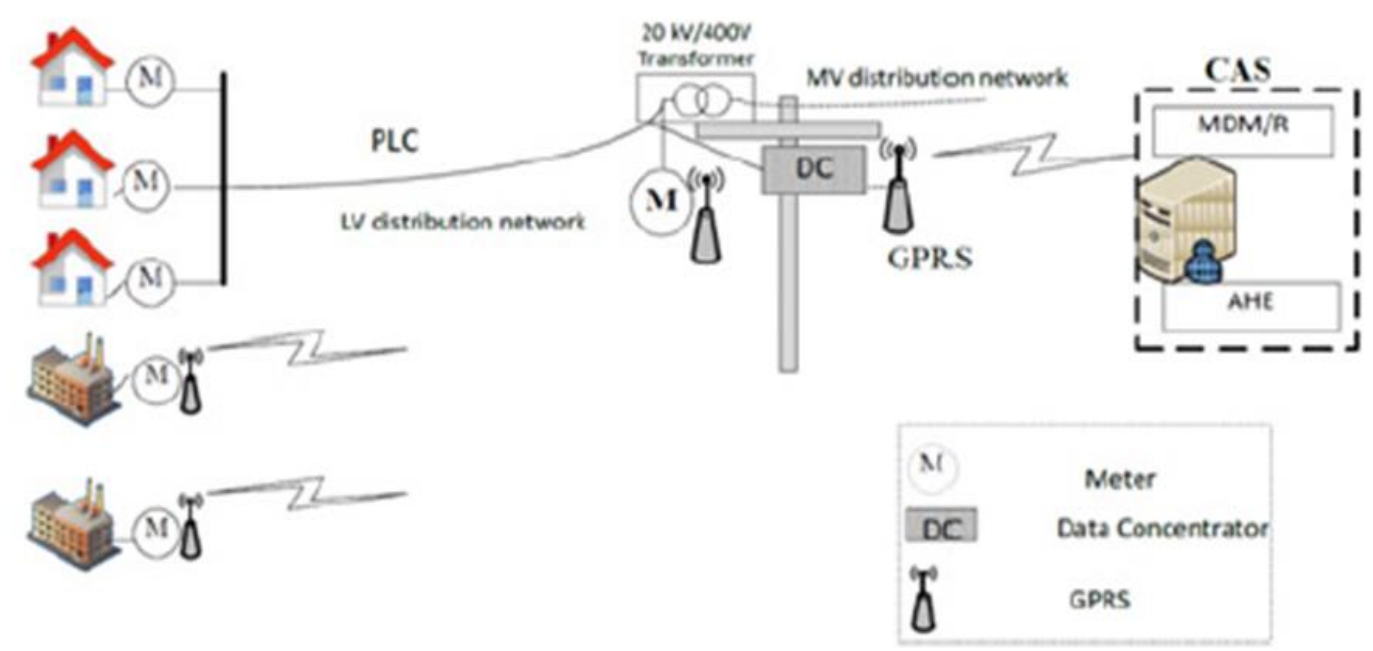

Fig. 4. Basic schema of smart metering with PLC

\section{PLC Metering Field Implementation}

This research was done in Yeşilırmak Distribution Company $250 \mathrm{kVA}$ distribution transformer residential area and Makel Company products was used to realize the field implementation such as mono phase meter, PLC Gateway, DC and Smart Metering software. One phase is enough to carry PLC signal that include meter data thus PLC method is same both three phase meter and mono phase meter. The investigation area grid is consist of overhead distribution lines. General view of Smart Metering with PLC research location is shown as Fig. 5.

Table 1. LV Aluminum rose conductor overhead distribution line parameters

\begin{tabular}{lcc}
\hline Properties & Unit & Value \\
\hline Current Carrying Capacity & $\mathrm{A}$ & $110-150$ \\
Resistance $(\mathrm{R})\left(20^{\circ} \mathrm{C}\right)$ & $\mathrm{Ohm} / \mathrm{m}$ & 1.354 \\
Conductor Cross Section (s) & $\mathrm{mm}^{2}$ & 21.14 \\
\hline
\end{tabular}

In addition, overhead distribution line technique parameters are given in table 1. Overhead distribution line consists aluminum featured rose conductor.

The $250 \mathrm{kVA}$ distribution transformer had four LV feeders to supply power own consumers. One of these feeders was chosen and SMs was installed to the residential consumer points. These SMs worked as transmit and receive point among each other and brought out NB-PLC mesh network thanks to existing LV distribution line. NB-PLC features were CENELEC A band, G3-PLC standard with 35.9 - 90.6 $\mathrm{kHz}$ frequency range via OFDM modulation technique for this field implementation [13-14]. G3-PLC has repetition code feature so error correction is executed efficient beside this G3-PLC is included robust mode that can send data four or six repeat [15-17]. Although PRIME standard data rate is higher than G3-PLC, G3-PLC is more powerful according to PRIME because robust and complete data is the most important thing in Smart Metering operation.

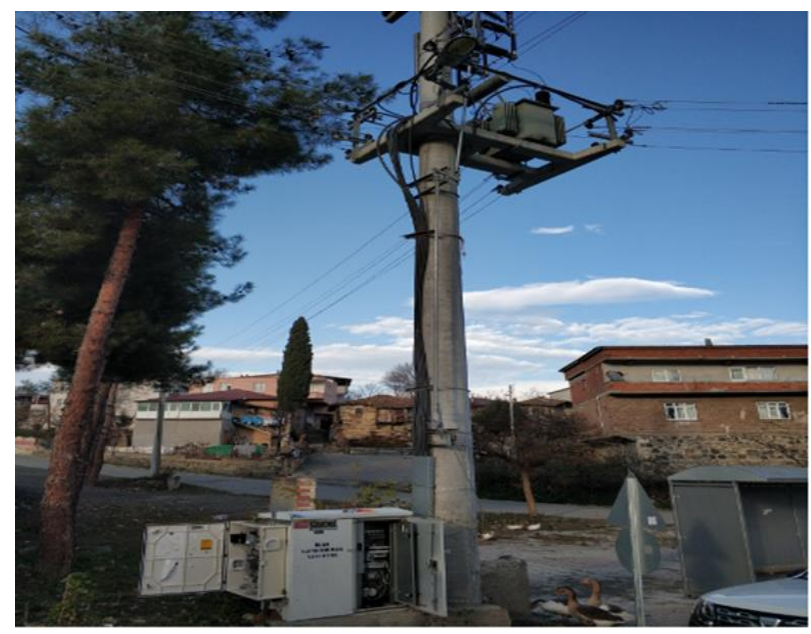

Fig. 5. General view of PLC research location

One of the key parameters of PLC is signal attenuation that related to SM and DC distance. The most important point is the nearest SM to DC where located in LV side of distribution transformer. If the distance is more than $35 \mathrm{~m}$ between DC and the closest SM to DC, The PLC will not provide any communication between meters because of attenuation for this field implementation area. This situation was experienced another LV distribution feeder consumer SM that had more than $35 \mathrm{~m}$ distance between DC.

SMs were located in Fig. 6. as red point via distance. These SMs succeed to reach their measurements to DC and DC sent their measurements to AMI software. This situation can be checked form software. Their register values are in AMI software according to their serial number. 


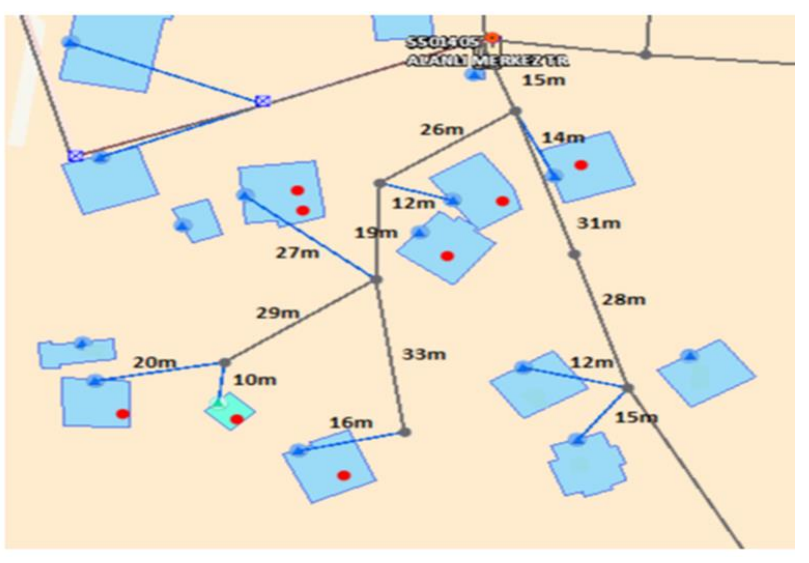

Fig. 6. Smart meters with NB-PLC locations in LV distribution grid

Meter and PLC gateway were installed externally each other by RS 485 communication port so SM has been obtained.

PLC Gateway
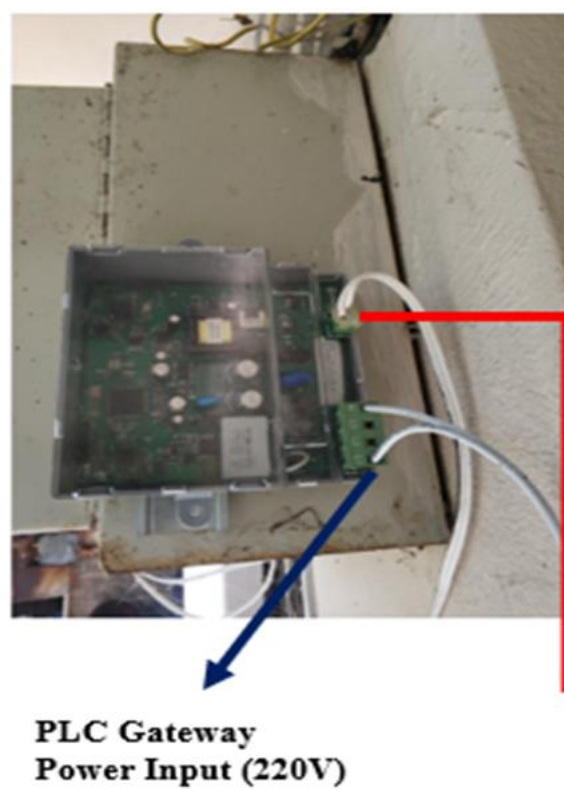

They were not combined in one box together for this field implementation but it is possible to design SM that can cover PLC gateway internally in one box. Implemented area consumers installations were explained in Fig. 7. Also, one PLC gateway can connect more than one meter because of RS 485 capability.

DC was located secondary part of transformer inside the low voltage distribution panel. It has installed with three phase connection to detect all SMs which were taken energy any phase. DC used GPRS connection to send all measured SMs data to the AMI software. GPRS sim card was plugged to DC communication module thus GPRS was used only one point, limited to DC for this field application. The connection of DC was pictured in Fig. 8.

\section{Mono phase Meter}

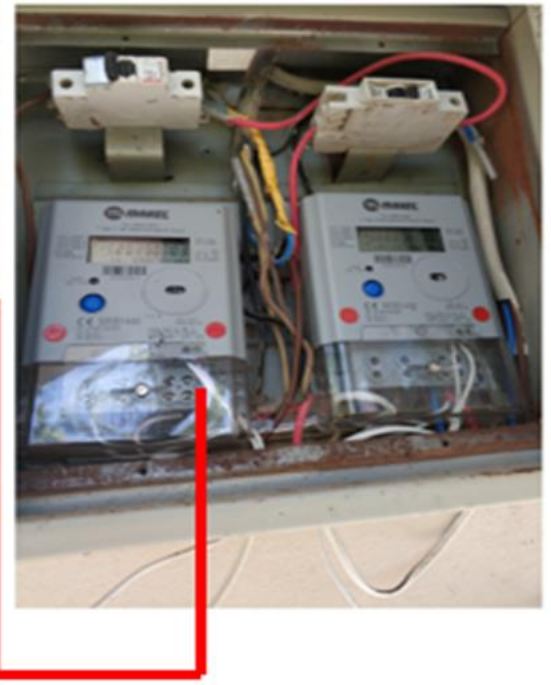

RS 485

Connection

Fig. 7. Connection between PLC gateway - meter in consumer installation

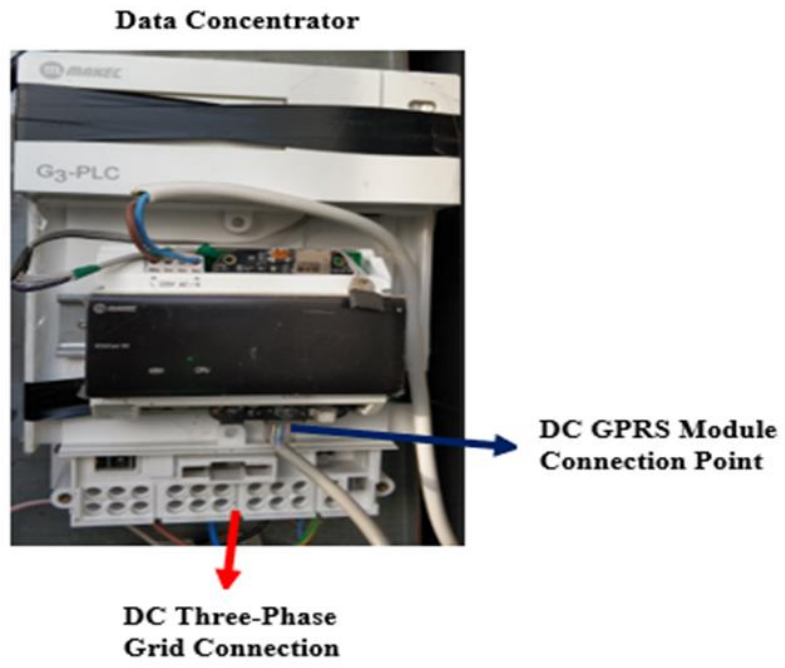

Fig. 8. Data concentrator type and connection
AMI systems main components were communication method, meter data management software, meter, gateway and DC for Smart Metering with NB-PLC field implementation. Meter and PLC gateway combination (Smart meter), DC and NB-PLC method has been introduced so far.

However only AMI software remains to promote. AMI software has captured consumers measured mono phase meter data thanks to DC by GPRS communication way for this research. AMI software has mainly two main parts one is central data center in background, other is end user interface in frontend. Captured measurement data that sent by DC, firstly evaluated according to software rule and business requirements and then it was transferred to end user screen. SMs that was used field implementation results which 
obtained via NB-PLC is shown in Fig. 8. that produced from AMI software end user side.

Active energy registers (1.8.0, 1.8.1, 1.8.2, 1.8.3 obis codes) and maximum power register (1.6.0 obis code) have been shown as example with their real measurement date from AMI software end user part with in field implementation. Beside this SM serial number and name seem to follow SM in software. It is possible to see some another meter reading results such as current-voltage information, phase outages, load profile and some alarm obis for the meter security.

\section{Results and Discussions}

AMI user main screen is shown in Fig. 9. This screen is included that general meter information like tariffs, meter reading time, meter serial number and also customizing and management tools are in there like reports, warning messages, gateway operations. Fig. 9. provides us meter real reading results sample with time stamp meter by meter. New samples can be generated easily for same meter in different times.

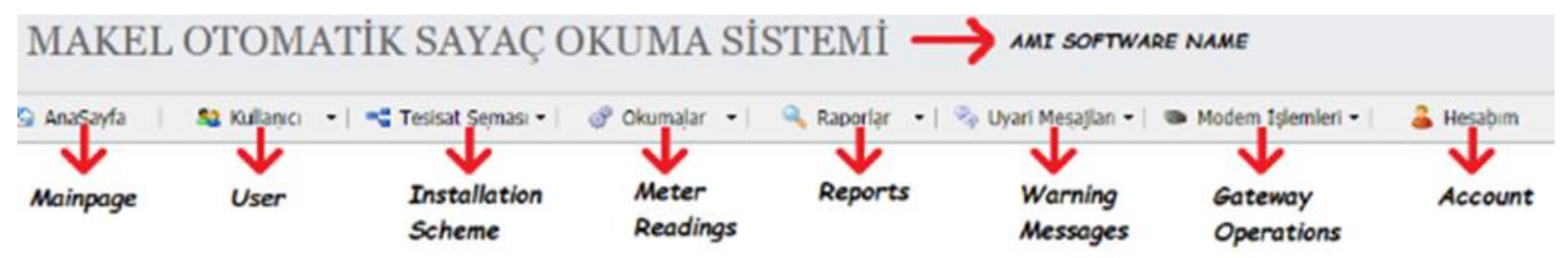

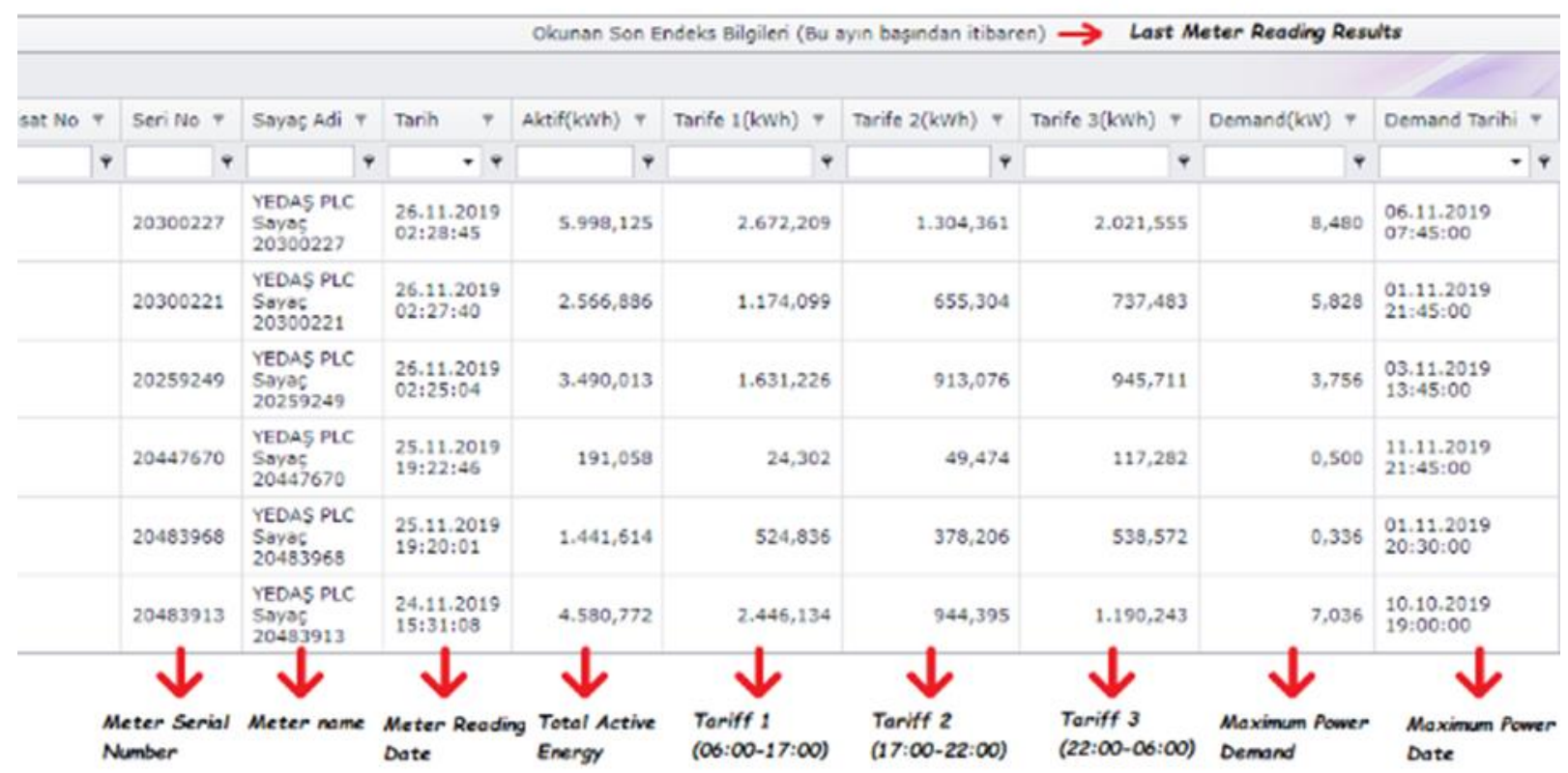

Fig. 9. Smart meter reading with NB-PLC results from AMI software

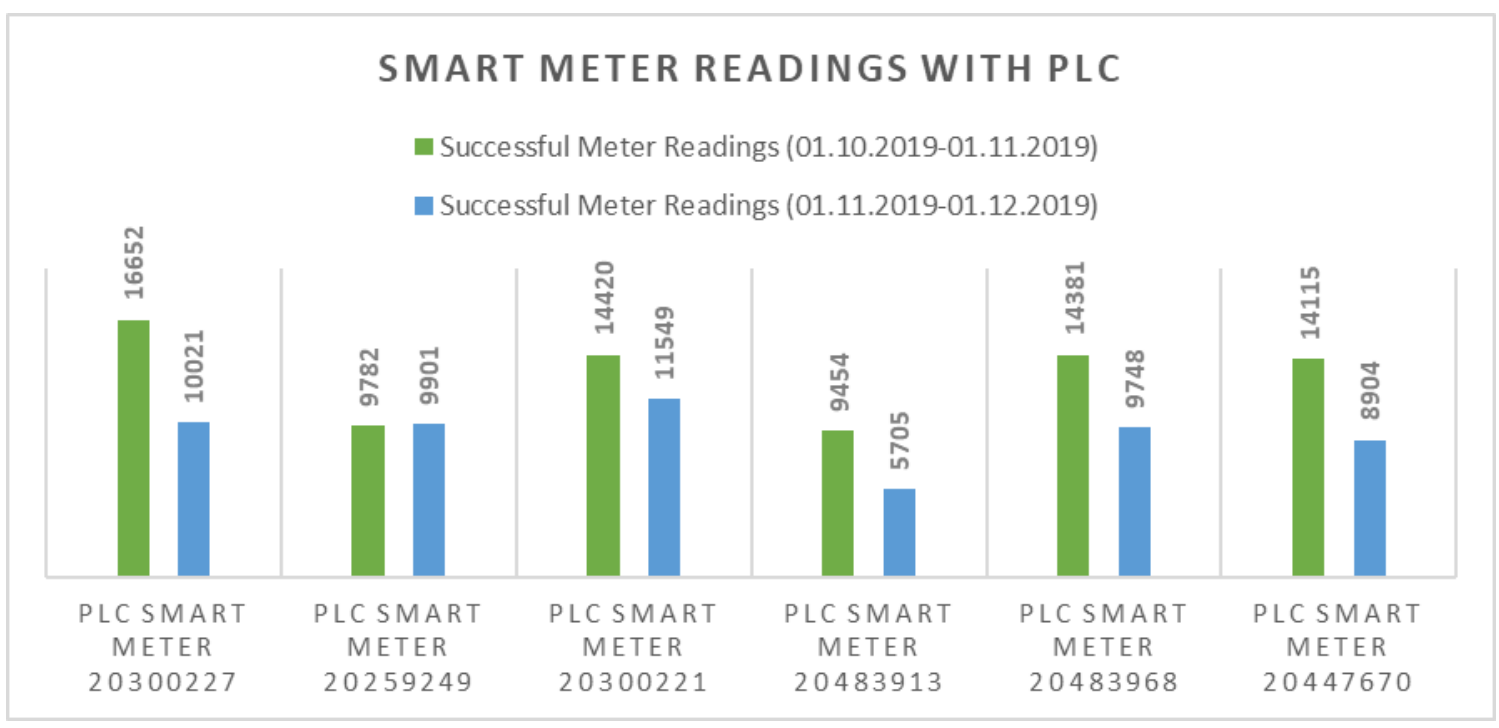

Fig. 10. Number of successful smart meter reading results with PLC 
In order to determine success of smart metering with NBPLC field investigation, SM successful meter readings results which were short readout, long readout, load profile and on demand readout were evaluated. These SM meter readings have been obtained from AMI software as a report. According to this report, accomplished SM readings numbers graphic was produced in Fig. 10.

\section{Conclusions}

The research of smart metering with NB-PLC was conducted in Yesilırmak Distribution Company field. The consumers with PLC featured SM were in the $250 \mathrm{kVA}$ distribution transformer residential area. G3-PLC standard was used for NB-PLC. Meter - PLC gateway field connection and DC field installation explained and measured data with NB-PLC was shown via AMI software in Fig. 8. Consumer side SMs communication are provided with NB-PLC successfully and GPRS has been only used one place to be DC. Thus, communication costs have been reduced and dependency on GSM companies has decreased for smart metering operations, also PLC-GPRS hybrid communication model has been revealed. On the other hand, increased switching number and impedance changes in the grid are the disadvantages of PLC. Successful meter reading with NBPLC results that is shown in Fig. 9. and Fig.10 are enough for distribution company to manage for residential area customers meter reading and billing operations. In addition, when PLC featured SMs install, the distance between DC and SMs should be considered to avoid signal attenuation that prevents meter reading with PLC. Distribution companies must research different communication way according to their field features except GPRS in order to manage smart metering operation more quantity, more successful, cheaper and more independent. This study shows that NB-PLC is one of the successful alternative communication way for smart metering process in distribution grid. As a future perspective, all details should be investigated whether SM data can be efficiently transferred from transformer to transformer by PLC method at medium voltage level.

\section{Acknowledgement}

This study has presented as oral at International Conference on Advanced Technologies (ICAT'20), and, it has been published at conference abstract book as abstract.

\section{ORCID}

M. Şen

S. Üstün Ercan

\section{References}

[1] Usman, A. and Shami, S.H. 2013. Evolution of communication technologies for smart grid applications. Renewable and Sustainable Energy Reviews, 19, 191-199.

[2] Zheng, J., Gao, D.W. and Lin, L. 2013. Smart meters in smart grid: an overview. 2013 IEEE Green Technologies Conference, 4-5 April, Denver, USA, 5764.

[3] Kabalci, Y. 2016. A survey on smart metering and smart grid communication. Renewable and Sustainable Energy Reviews, 57, 302-318.

[4] Zhou, X., Ma, Y., Gao, Z. and Wang, H. 2017. Summary of smart metering and smart grid communication. 2017 IEEE International Conference on Mechatronics and Automation, 6-7 August, Takamatsu, Japan, 300-304.

[5] Yaqoob, I., Hashem, I.A.T., Mehmood, Y., Gani, A., Mokhtar, S. and Guizani, S. 2017. Enabling communication technologies for smart cities. IEEE Communications Magazine, 55(1), 112-120.

[6] Lopez, G., Matanza, J., De La Vega, D., Castro, M., Arrinda, A., Moreno, J.I. and Sendin, A. 2019. The role of power line communications in the smart grid revisited: applications, challenges, and research initiatives. IEEE Access, 7, 117346 - 117368.

[7] Yigit, M., Gungor, V.C., Tuna, G., Rangoussi, M. and Fadel, E. 2014. Power line communication technologies for smart grid applications: a review of advances and challenges. Computer Networks, 70, 366-383.

[8] Sharma, K. and Saini, L.M. 2017. Power-line communications for smart grid: progress, challenges, opportunities and status. Renewable and Sustainable Energy Reviews, 67, 704-751.

[9] Sadowski, Z. 2015. Comparison of PLC-PRIME and PLC-G3 protocols. 2015 International School on Nonsinusoidal Currents and Compensation, 15-18 June, Lagow, Poland, 1-6.

[10] Lisowski, M., Masnicki, R. and Mindykowski, J. 2019. PLC-enabled low voltage distribution network topology monitoring. IEEE Transactions on Smart Grid, 10(6), 6436-6448.

[11] Foudeh, H.A. and Mokhtar, A.S. 2015. Automated meter reading and advanced metering infrastructure projects. 2015 9th Jordanian International Electrical and Electronics Engineering Conference, 12-14 October, Amman, Jordan, 1-6.

[12] Khazaee, A., Safa, H.H., Ghasempour, M. and Delavari, H. 2017. Distribution loss reduction in residential and commercial pilots by using AMI system. 
CIRED - Open Access Proceedings Journal, 2017(1), 1711-1714.

[13] Mlynek, P., Misurec, J., Kolka, Z., Slacik, J. and Fujdiak, R. 2015. Narrowband power line communication for smart metering and street lighting control. IFAC-PapersOnLine, 48(4), 215-219.

[14] Borovina, D., Zajc, M., Mujcic, A., Tonello, A. and Suljanovic, N. 2020. Error performance analysis and modeling of narrow-band PLC technology enabling smart metering systems. International Journal of Electrical Power \& Energy Systems, 116, 1-10.

[15] Hoch, M. 2011. Comparison of PLC G3 and PRIME. International Symposium on Power Line Communications and Its Applications, 3-6 April, Udine, Italy, 165-169.

[16] Masood, B. and Baig, S. 2016. Standardization and deployment scenario of next generation NB-PLC technologies. Renewable and Sustainable Energy Reviews, 65, 1033-1047.

[17] Slacik, J., Mlynek, P., Fujdiak, R. and Dobesava, K. 2017. Experimental measurements of multi-carrier power line communication systems. 40th International Conference on Telecommunications and Signal Processing (TSP), 5-7 July, Barcelona, Spain, 91-96.

[18] Ozgonenel, O., Thomas, D.W.P. and Ercan, S.U. 2017. Power line communication through distribution transformers in smart grid. 40th International Conference on Telecommunications and Signal Processing (TSP), 5-7 July, Barcelona, Spain, 255-258.

[19] Ercan, S.U., Ozgonenel, O., Haj, Y.E., Christopoulos, C. and Thomas, D.W.P. 2017. Power line communication design and implementation over distribution transformers. 10th International Conference on Electrical and Electronics Engineering (ELECO), 30 November-2 December, Bursa, Turkey, 190-194.

[20] Ercan, S.U., Ozgonenel, O. and Thomas, D.W.P. 2018. Power line communication channel for smart grid. 6th International Istanbul Smart Grids and Cities Congress and Fair (ICSG), 25-26 April, Istanbul, Turkey, 208212.
NOTE: This article was presented at International Conference on Advanced Technologies (ICAT'20). It was evaluated by 2 Reviewers before publish, and it has been passed all publication and evaluation processes of journal. 